\title{
Machine Learning for detection of viral sequences in human metagenomic datasets
}

\author{
Zurab Bzhalava ${ }^{1 \dagger}$, Ardi Tampuu $^{2 \dagger}$, Piotr Bała $^{3}$, Raul Vicente ${ }^{2}$ and Joakim Dillner ${ }^{1 *}$
}

\begin{abstract}
Background: Detection of highly divergent or yet unknown viruses from metagenomics sequencing datasets is a major bioinformatics challenge. When human samples are sequenced, a large proportion of assembled contigs are classified as "unknown", as conventional methods find no similarity to known sequences. We wished to explore whether machine learning algorithms using Relative Synonymous Codon Usage frequency (RSCU) could improve the detection of viral sequences in metagenomic sequencing data.

Results: We trained Random Forest and Artificial Neural Network using metagenomic sequences taxonomically classified into virus and non-virus classes. The algorithms achieved accuracies well beyond chance level, with area under ROC curve 0.79. Two codons (TCG and CGC) were found to have a particularly strong discriminative capacity.

Conclusion: RSCU-based machine learning techniques applied to metagenomic sequencing data can help identify a large number of putative viral sequences and provide an addition to conventional methods for taxonomic classification.
\end{abstract}

Keywords: Machine learning, Metagenomic sequencing, Human samples, Viral genomes

\section{Background}

A large number of different viruses are present in biospecimens from humans $[1,2]$. The proportion of viral sequences and its composition seem to change in diseased individuals $[3,4]$. As many novel viruses are continuously discovered, it is possible that many human viruses are yet to be detected [5-10]. Next Generation Sequencing (NGS) technologies are used to directly examine the DNA present in clinical samples, without the requirement of prior information about sequences that may be present [11]. Metagenomics refers to the complete sequencing of all microbiological genomes in a biospecimen and viral metagenomics is routinely used for virus detection and discovery of new viruses [5, 9, 10, 12-17]. In order to detect potential viral sequences in metagenomic datasets, conventional alignment-based classification is performed by BLAST, which compares sequences to known genomes and calculates how much similarity they share. A downside of the method is that public

\footnotetext{
*Correspondence: joakim.dillner@ki.se

†Zurab Bzhalava and Ardi Tampuu contributed equally to this work.

${ }^{1}$ Dept. of Laboratory Medicine, Karolinska Institutet, F46, Karolinska University Hospital Huddinge, Stockholm, Sweden

Full list of author information is available at the end of the article
}

databases for virus-related genomes are incomplete. A large number of sequences are labeled as "unknown" since many of them have only very distant or no homologs in public databases $[5,7]$.

The HMMER3 algorithm implements Hidden Markov Models with a reference set of sequences encoding viral proteins ("vFams") [18]. This method appears to be more effective in detecting distant homologs in metagenomic datasets [19]. However, it is also dependent on a reference database such as "vFams", which like any other public database is incomplete. Predictive models (for example built via machine learning algorithms), on the other hand, use a training database only to learn what the relevant features and criteria for classification are, and can then be applied to any new data point. In particular, we propose that machine learning methods as presented in our work can act as a recommendation system to sort and prioritize the sequences marked as "unknown" by existing methods for further research.

In this work, we wished to investigate whether machine learning using the relative synonymous codon usage (RSCU) in the sequences could be used to predict the

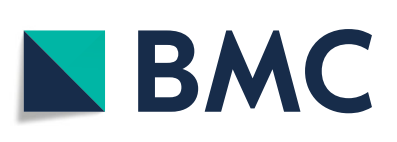

(c) The Author(s). 2018 Open Access This article is distributed under the terms of the Creative Commons Attribution 4.0 International License (http://creativecommons.org/licenses/by/4.0/), which permits unrestricted use, distribution, and reproduction in any medium, provided you give appropriate credit to the original author(s) and the source, provide a link to the Creative Commons license, and indicate if changes were made. The Creative Commons Public Domain Dedication waiver (http://creativecommons.org/publicdomain/zero/1.0/) applies to the data made available in this article, unless otherwise stated. 
presence of human virus sequences in metagenomic NGS data. In the genetic code, some amino acids are encoded by several, synonymous codons. Usage of these codons is not random and differs among species. This phenomenon is called Codon Usage Bias. Several viral families (in particular the herpesvirus, lentivirus, papillomavirus, polyomavirus, adenovirus, and parvovirus) are known to encode structural proteins that display heavily skewed codon usage compared to the host cell [20,21].

In order to test whether codon usage could predict viral nature of a sequence we used genes extracted from NCBI GenBank to build a virus/non-virus classifier. A cross-validation approach using Random Forests achieved almost perfect accuracy on this dataset. However, the models trained on NCBI GenBank data fail to generalize to classifying contigs obtained from metagenomics analysis, with random-like performance. As the main contribution, we trained Random Forests and neural networks on a metagenomic sequencing dataset generated by NGS technologies applied to human biospecimens. As we wished to develop an algorithm to detect presence of human viruses, viruses infecting bacteria (phages) were not included in the training set. We show that models trained using RSCU values from contigs from a set of metagenomics experiments generalizes to other metagenomics experiments. Furthermore, we investigated which codons were more important for the models to classify a sequence as a virus.

\section{Methods \\ Dataset \\ Patients and samples}

Next Generation Sequencing (NGS) using the Illumina platform was used to generate the metagenomic sequencing datasets from human samples coming from several different patients groups, as described [6-8, 22-24]. The purpose of all of these studies was to investigate the presence of viral genomes or other microorganisms in human biospecimens from patients who developed diseases or from matched control subjects. Further information of the samples is provided in Additional file 1.

\section{Sequencing}

Sequences were generated from the MiSeq, NextSeq and HiSeq (Illumina) sequencing platforms, as described by the manufacturer. When multiple human samples were included in the same sequencing run, the sequences were mapped to the originating sample using sequence indices, included in the Illumina adapters.

\section{Bioinformatics pipeline}

Before applying machine learning techniques, all sequencing experiments were analyzed using a benchmarked bioinformatics pipeline, as described [25]. The pipeline starts with quality checking and reads are trimmed according to their Phred quality scores. After this, reads that are highly similar (with 95\% identity over $75 \%$ of their length) to human, phage, bacterial and vector DNA are removed from further analysis using BWA-MEM [26]. The rest of the reads are normalized and then processed for assembly using the Trinity [27], SOAPdenovo, SOAPdenovo-Trans [28] and IDBA-UD [29] assemblers. we used several assembly algorithms in order to validate results. Then the assembled contigs are subjected to taxonomic classification using alignmentbased classifiers such as BLAST and HMMER3. The code of the pipeline is available on GitHub (https://github. com/NIASC/VirusMeta and https://github.com/NGSeq/ ViraPipe). Different steps of the pipeline are shown in Fig. 1.

\section{Feature extraction and labeling}

Sequencing datasets were obtained from 19 different NGS experiments. After the de novo genome assembly, two different algorithms were applied for viral classification. Firstly BLASTn algorithm (reward for nucleotide match $=1$; penalty of nucleotide mismatch $=1$; cost to open a gap $=0$; cost to extend a gap $=2$; e-value $\leq e^{-4}$ ) was applied with NCBI nucleotide database. We also re-analysed the assembled contigs by PCJ-BLAST [30] with the most recent version of nt database. Number of contigs classified by BLAST into different taxonomy groups is shown in Additional file 2. For the contigs that were classified as unknown we used HMMER3 algorithm. As reference database for this algorithm, we used a database which includes viral profile hidden Markov models ("vFams") from all the virally annotated proteins in RefSeq (http://derisilab.ucsf.edu/software/vFam) [18]. Both BLAST and HMMER3 results were used for the machine learning. Note that while BLAST classifies sequences in different taxonomic groups, HMMER3 with the "vFam" reference set only identifies viral genomes. All assembled-sequences, classified and labeled by this bioinformatics pipeline were combined to train the machine and deep learning algorithms. This dataset consisted of $3 \%$ of viral contigs. Usually, viruses are less than $0.1 \%$ in a metagenomic dataset but the removal of highly identical non-viral reads at the initial stage of the analysis relatively increases the proportion of viruses in the dataset.

To extract features from the metagenomic dataset for the machine learning purposes we used Relative Synonymous Codon Usage frequency (RSCU) [31]. Proteins are encoded by 20 different amino acids but there are 64 codons encoding for them. Trinucleotides coding for the same amino acids are called synonymous codons and usage of those is not random: some species prefer one codon over another. For a given contig, we calculate the RCSU value for each codon with the following formula: 


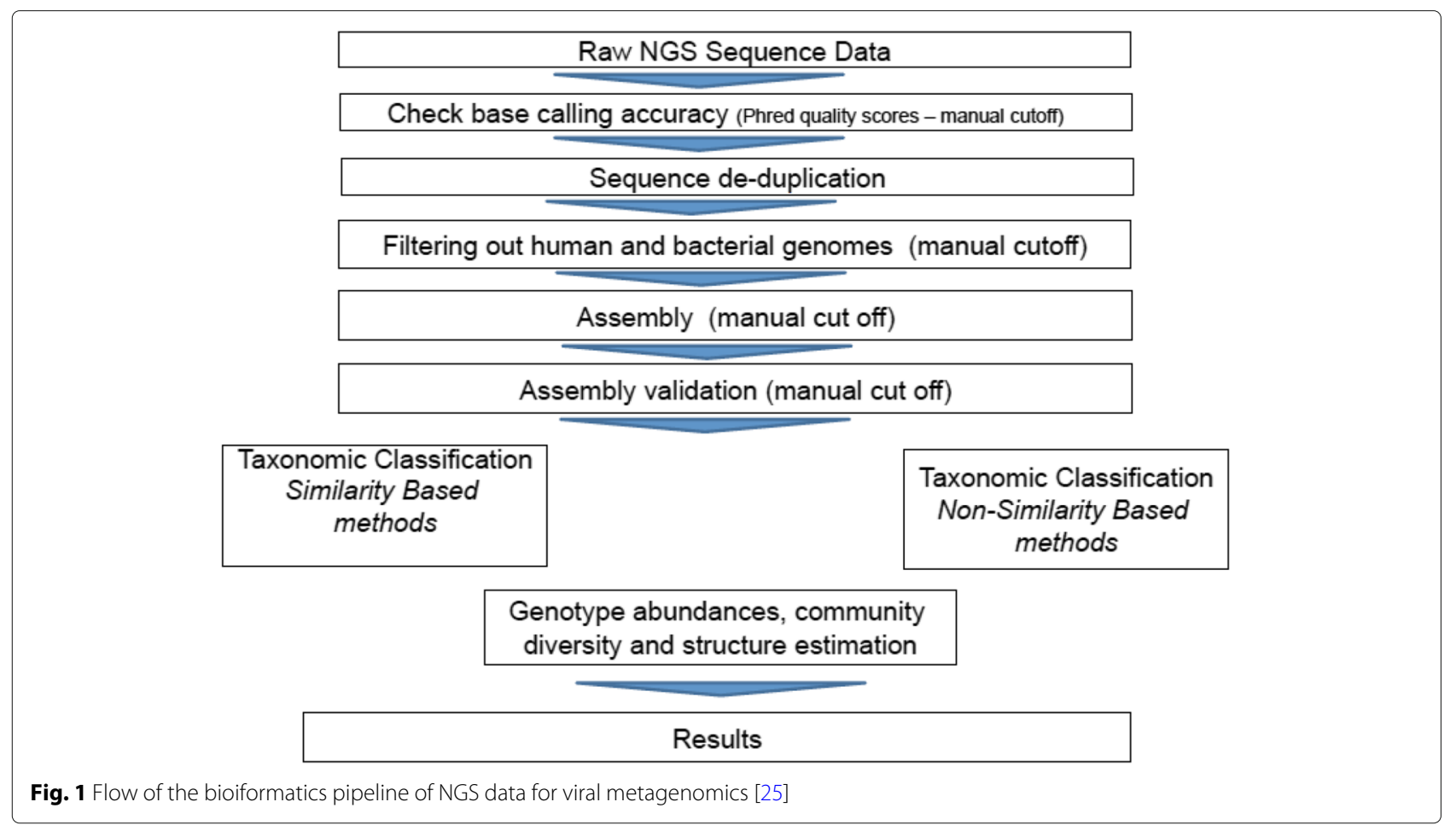

$$
f_{i j}=\frac{x_{i j}}{\frac{1}{n_{i}} \sum_{j=1}^{n_{i}} x_{i j}}
$$

Where $x_{i j}$ is the number of occurrences of $j$-th synonymous codon coding for amino acid $i . n_{i}$ is the total number of synonymous codons that encode for amino acid $i$. This ratio can be defined as the observed number of codon occurrences divided by expected usage assuming uniform distribution [31]. Methionine and Tryptophan that have only one corresponding codon (ATG and TGG, respectively) were removed from the analysis since they would not contribute to the study. Furthermore, stop codons were also removed. This gave us a total number of 59 features.

DNA has double strands: forward (5 to 3') and reverse (3 to $\left.5^{\prime}\right)$. Since in metagenomic sequencing data it is not known from which strand a contig came from, we counted RSCU values for both directions and considered them as two independent samples. Furthermore, because of the fact that RSCU values are counted only in regions of Open Reading Frames (ORFs), assembled-sequences that did not have at least two ORFs in either direction were discarded from the further analysis. In this study, a stretch of codons was considered as an ORF if there was a stretch of at least 120 nucleotides between a start codon and a stop codon.

\section{Dataset from NCBI GenBank}

The NCBI GenBank sequences were obtained from Codon Usage Database (https://www.kazusa.or.jp/codon/). For this database codon usage (RSCU values) was calculated for complete genes using nucleotide sequences from the Genbank. For the analysis we used approximately 600 thousand proteins from which $14 \%$ were viral.

\section{Machine learning analysis}

In the preliminary analysis using Genbank data we first took a 10-fold cross validation approach using Random Forests with different sizes and with/without balancing the class weights. The reported results are the validation performance from the best performing parameter configuration, averaged over the 10 folds. Secondly we trained a Random Forest model on the entire NCBI GenBank dataset and tested its performance on RSCU values from contigs obtained from metagenomics experiments.

In the main body of work we trained models using metagenomics datasets. We used assembled-sequence data from 19 different metagenomic sequencing runs. However, we did not combine all contigs into one big dataset, because contigs from the same run might be highly similar to each other (in terms of hamming distance, for example). We applied cd-hit-est algorithm [32] with sequence identity threshold 0.98 and coverage 0.95 on the entire dataset to remove highly similar assembledsequences. However, we observed that contigs from the same run are still more likely to be similar than contigs from different runs. We did not want such highly similar sequences to end up in both training and testing data, because it would result in an artificially high 
accuracy that does not reflect the true ability to generalize to unseen samples. In order to provide an honest estimate of the test accuracy, we took an approach we call leaveone-experiment-out cross-validation (LOEO). With this approach, we trained our machine learning algorithms on data coming from 18 metagenomic sequencing runs and tested them on the remaining 19th run. We repeated this process 19 times and each time data from a different experiment was used as test set. Using this methodology we test algorithms on truly unseen data from an entirely different experiment, which gives us a fairer estimate of the performance of the model compared to a traditional K-fold cross validation.

The leave-one-experiment-out cross-validation approach gave us 19 different models and 19 validation sets of different size and prevalence. In order to combine results from different folds, we used macro and micro averaging approaches [33]. In macro averaging, the number of samples in each validation set is disregarded and the 19 results are simply averaged [33] (we average over datasets). In the micro averaging approach, however, experiments that provide more validation samples have a bigger influence on the results [33] (we average over samples).

In this study, we applied this leave-one-experiment-out approach using two classification algorithms: the main results were obtained using Random Forests, but later we validated the results with Artificial Neural Networks. We have selected to use Random Forest and Neural Networks for several practical and theoretical reasons. Most importantly, the hierarchical structure of the two algorithms allows combining the relatively simple features, such as RSCU values, to form more complex decision boundaries than simple non-linear regression models or SVMs. In addition, the capacity of both algorithms is easily controllable and they are widely used yielding state of the art results in many tasks.

\section{Random forest}

Random Forest is a collection of a large number of decision trees. Each tree differs from others because it is trained on a different set of training samples and because at each splitting point only a random subset of features are considered [34]. The differences between the trees work together and the average prediction made by the group is more accurate than one individual tree [34]. In this study, we used scikit-learn-0.18.1 implementation in python 2.7 [35]. More thorough description of Random Forests and the hyperparameters we tested is given in Additional file 3.

Feature importance in random forest Each decision tree in a random forest is a collection of simple splitting rules (if-then statements) that use only one feature at a time. At each splitting point only a small subset of features are considered. Among the possible one-feature if-then statements, the rule that maximally reduces Gini impurity is always chosen. Gini impurity is reduced if the two nodes resulting from the rule have a less uniform class distribution than the parent node. How important a feature was in an entire tree can be estimated by summing up the impurity reductions brought about by this feature at all different branching points where it was used [34, 36]. The importance of each feature in each individual tree is calculated and easily accessible to the user in scikit-learn's RandomForestClassifier [35].

To compare the importance of RSCU values of different codons for our classification task, we averaged the importance of the features across 1000 trees trained on the entire data set of all 19 experiments. When interpreting the mean importance of features, we need to notice that the RSCU values of synonymous codons can be highly correlated - if the value for one synonymous codon is high, the other(s) must be low. If there are only two synonyms, the correlation is almost perfect. Correlated features "compete" for importance - the RSCU value that is used first in a given tree will have the chance to contribute the information shared between the correlated features and is likely to show up as more important $[36,37]$. In a different tree the randomness might lead to another feature being selected first and contributing highly. This leads to high variance of feature importance across trees. Despite high variance, we believe the average importance is still interpretable and reveals which codons' RSCU values are useful more often than others [36], especially when the differences are clearly visible.

\section{Artificial neural networks}

Artificial Neural Networks is a machine learning algorithm inspired by the structure of the biological networks of neurons in the brain. The simplest type of artificial neural networks, feed-forward neural network (FFN) used in this work, consists of multiple layers of nodes (called "neurons") and the connections between these nodes [38]. There are no connections between the nodes of the same layer, whereas neighboring layers are all-to-all connected with each other. Each node is characterized by its activation and each connection by its weight. These connection weights can be optimized by providing the network inputoutput pairs.

To implement neural network models we used version 2.0.5 of Keras library (https://keras.io/) in Python 2.7. The procedure to find useful values for hyperparameters (network size, depth, etc) and a more thorough description of neural network approach is provided in Additional file 3.

\section{Results}

To test whether the relative synonymous codon usage frequency can predict the viral nature of a sequence we firstly trained a model on sequences originating 
from NCBI GenBank. Secondly, we trained models only on assemebled metagenomics contigs. We show how well these models classify viral sequences and compare results. During this study we used two datasets and in each dataset there was a high class imbalance. Therefore, we used area the under Receiver Operating Characteristic (ROC) curve as our main metric to evaluate the results since it is not dependent on class distribution.

We start with results from a preliminary analysis obtained using the dataset from NCBI Genbank. Thereafter, in the main analysis, we first describe and analyze the results obtained on metagenomics datasets with Random Forest (RF) classifier. Also, we analyze how important role each RSCU value played in the classification. Finally, to validate the results obtained with RF, we demonstrate that a similar classification performance can be achieved with feedforward neural networks.

\section{GenBank model}

The first set of random forest models was trained on RSCU values obtained from genes registered at Genbank. Using $k$-fold cross validation method $(\mathrm{k}=10)$ the approach showed very high accuracy. Figure 2 shows that averaged across all folds the random forests achieve 0.99 area under the ROC curve, meaning that classifying genes based on their RSCU values can be done almost perfectly. As a next step we applied a model trained on GenBank data on metagenomics data in order to see how well it would generalize on this type of noisy dataset. As Fig. 3 shows the model clearly failed to classify the assembled metagenomics contigs. The area under the ROC curve was 0.51 . Despite the fact that the same model performed very well on a dataset obtained from Genbank its accuracy on metagenomics was very close to a random classifier.

\section{Metagenomics model}

As our primary goal was to detect viral sequences in metagenomics and given the fact that Genbank model could not classify assembled contigs, we trained the next model entirely on metagenomics data.

We used Random Forests with different hyperparameter combinations (number or trees, up and down sampling, class weights), applying the leave-one-experiment-out (LOEO) type cross-validation (see "Methods" section). The results with different tested hyperparameters are provided in Additional file 4. The best results were obtained with 5000 trees, balanced class weights and no up or down sampling. In the following we present the results from this model, unless clearly stated otherwise.

Joining the predictions for all samples and for all experiments in our LOEO cross-validation approach, we can describe the overall performance of the random forests. In Fig. 4 we visualize the ROC curves for each individual validation set (i.e. data from each of the 19 metagenomics experiments, dotted gray lines) as well as the micro-averaged and macro-averaged ROC curves across the 19 validation sets (blue line

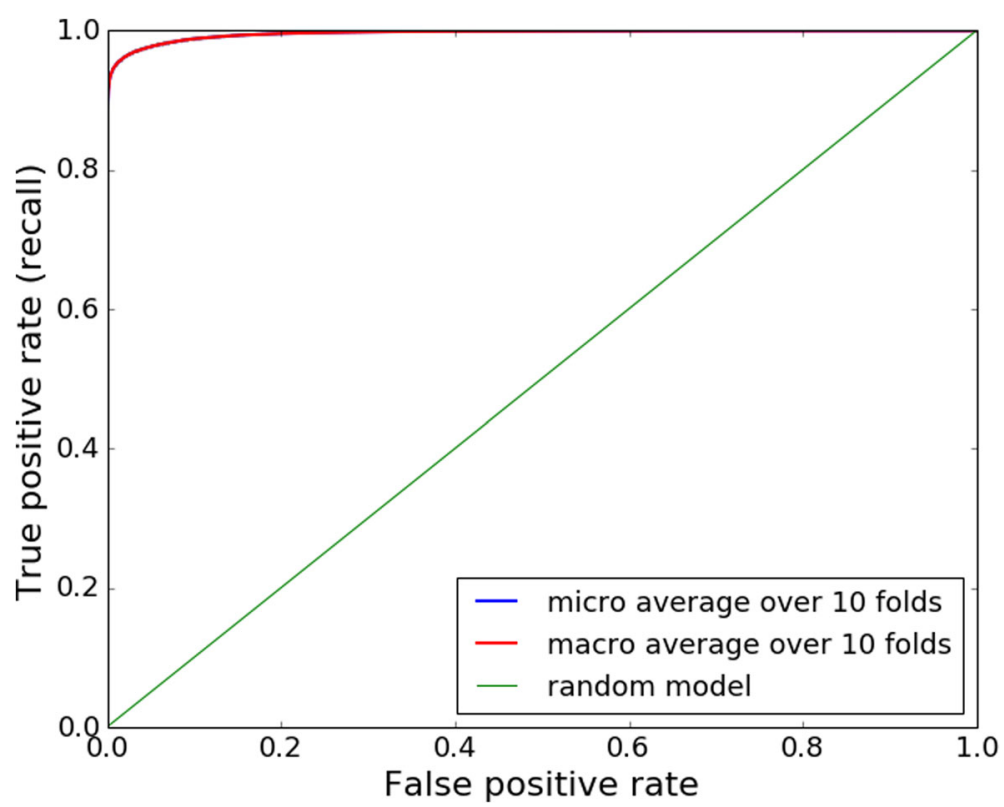

Fig. 2 ROC curve of GenBank model when testing on GenBank data. The models trained in 10-fold cross validation, achieved 0.99 area under the ROC curve while classifying RSCU values obtained from genes registered at the GenBank. Notice that in case of equal fold sizes, micro and macro averages are equal 


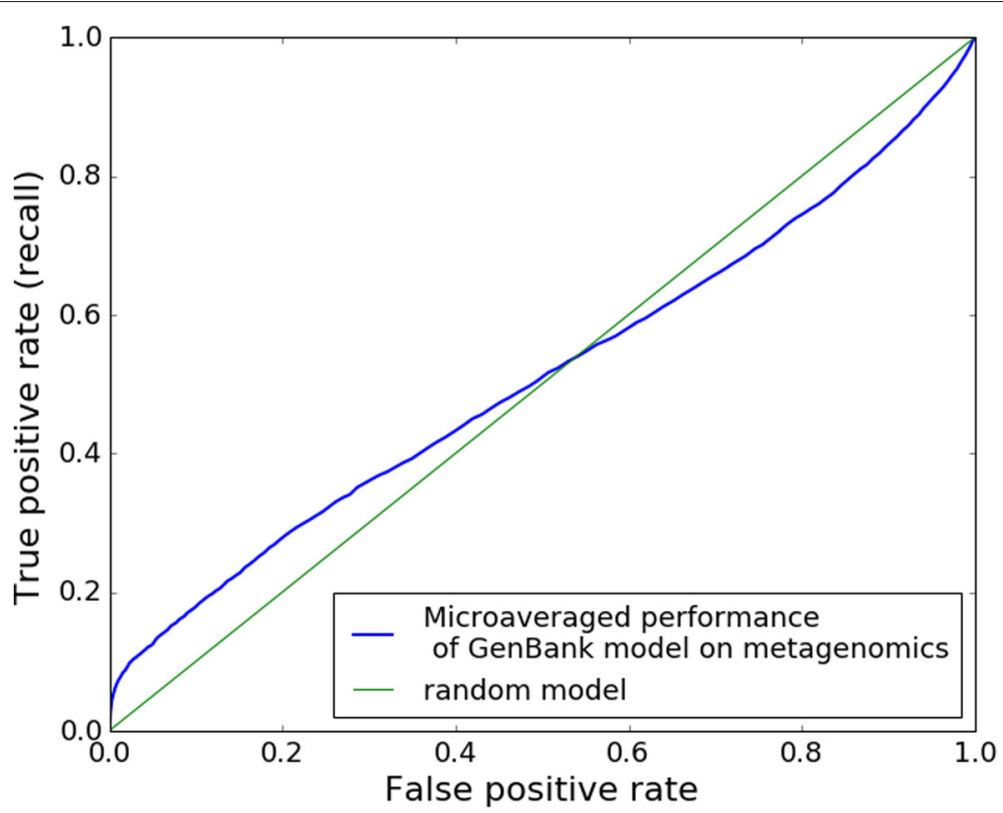

Fig. 3 ROC curve of GenBank model when tested on metagenomics data. Area under the ROC curve is 0.51 , which means the model trained on GenBank data failed to generalize on metagenomics data. It's performance is close to random level

and red line respectively). The area under the microaveraged ROC curve is 0.789 meaning the models performed clearly better than a random classifier or the Genbank model. Because our data comes from very different experiments, we also provided the macroaveraged results - statistics from all 19 cross-validation folds are averaged disregarding the number of samples. The area under the macro-averaged ROC curve is 0.785 , confirming that the models perform well on data from different experiments. See Additional file 5 for results per validation set (i.e per metagenomics experiment).

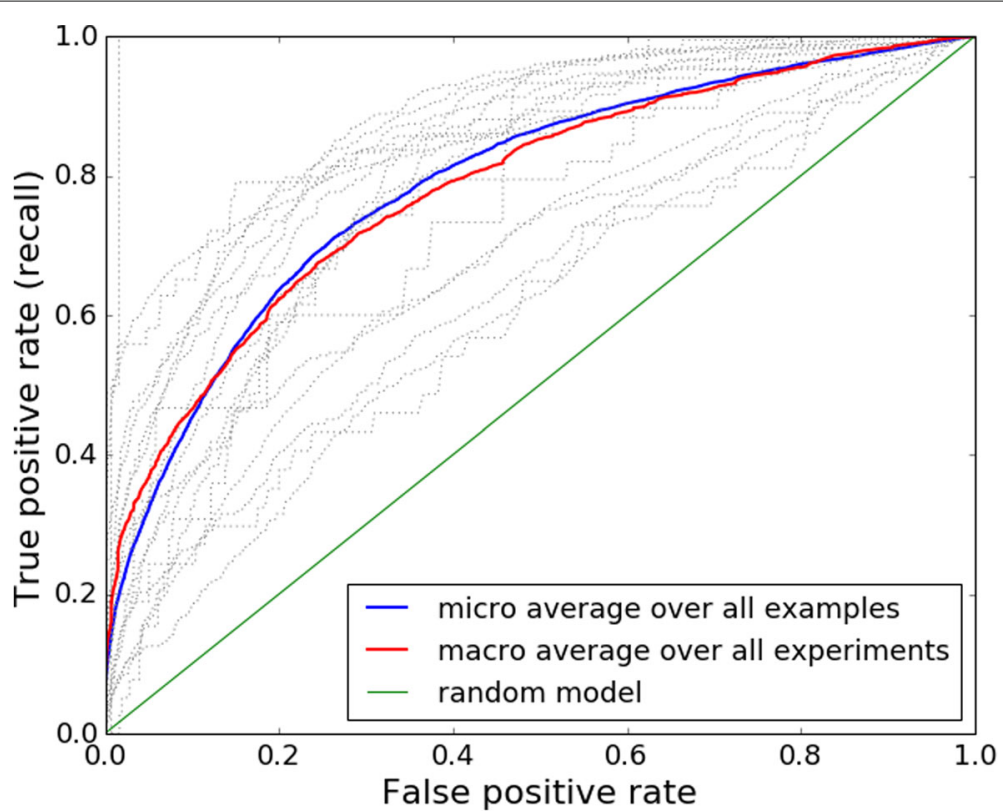

Fig. 4 ROC curves of the metagenomics model for each LOEO cross-validation fold and their micro and macro averages. The grey lines depict the ROC curve in each leave-one-experiment-out cross-validation fold. The red line is the macro-averaged curve and blue line the micro-averaged curve. Green line shows the performance of a random model. The averaged results are clearly above the diagonal, meaning the models perform a much better than a random guess 


\section{Precision and recall}

The leave-one-experiment-out (LOEO) cross-validation approach yields a probability for each contig being a virus. Across the 19 datasets, we had on average $3 \%$ of virus and $97 \%$ of non-virus samples. Notice that a naive model that classifies everything as non-virus would have a $97 \%$ overall precision and $97 \%$ overall recall. Despite rather high overall precision and recall, this model would clearly be useless for separating the classes. With a high class imbalance we needed to describe the precision and recall for both classes separately, instead of overall performance. This way we could gain more insight to the model's actual ability to detect viral samples. Table 1) summarizes the precision and recall at threshold $\mathrm{p}$ (virus) $\geq 0.5$ for the two classes, using micro and macro averaging. We see that the model is clearly doing better than simply classifying all samples as "not virus".

In Table 1 we reported the precision and recall if all samples with $\mathrm{p}$ (virus) $\geq 0.5$ are classified as virus. This is an intuitive threshold to set - it means that we assign the most probable class to each sample. Notice however, that setting a higher threshold would make the decision stricter and would likely increase the precision at the expense of recall, whereas a lower threshold boosts recall at the expense of precision. Varying the strictness of the classification can be useful depending on the context and purpose of the analysis. If one needs to detect the maximum amount of viruses and is willing to accept many false positives, a low threshold can be useful. Inversely, if false positives are costly to deal with while one can accept letting many viruses pass unnoticed, a high threshold might be useful. This is also the case in metagenomics analysis for finding new viral sequences - setting a threshold yielding high precision might be useful as further biological analysis can be costly. Figure 5 illustrates the trade-off between precision and recall for the virus class using the model with highest area under ROC curve. With this model we can, for example, achieve $75 \%$ accuracy at $8.0 \%$ recall, $90 \%$ accuracy at $5.6 \%$ recall and $95 \%$ accuracy at $3.7 \%$ recall. However, if choosing the model maximizing these recall

Table 1 Micro- and macro-averaged performance measures across 19 experiments using random forests

\begin{tabular}{lllll}
\hline Method & Class & Precision & Recall & F1-score \\
\hline Micro-average & Non virus & 0.97 & 1.00 & 0.99 \\
Micro-average & Virus & 0.92 & 0.05 & 0.10 \\
Macro-average & Non virus & 0.96 & 1.0 & 0.98 \\
Macro-average & Virus & 0.53 & 0.04 & 0.08 \\
\hline
\end{tabular}

The results presented are from the best model according to area under ROC curve $\left(R O C_{\text {micro }}=0.789\right)$. This model used 5000 trees, balanced class weights and no down nor upsampling values instead of maximizing area under ROC curve, we can also achieve $75 \%$ accuracy at $10.5 \%$ recall, $90 \%$ accuracy at $8.6 \%$ recall, $95 \%$ accuracy at $5.5 \%$ recall. See Additional file 4 for results with different hyperparameter values.

\section{Feature importance and visualization}

The mean importance of each codon's RSCU value for the virus detection task is displayed in Fig. 6.

The high variance in these mean importance values is driven by the correlations between the RSCU values of synonymous codons (see the explanation in "Methods" section). Despite the variance, it is clear that the sum importance of an amino acid seems to grow with increased number of triplets coding for it. Secondly, it can be seen that in most cases the importance is distributed rather uniformly across synonymous codons, but codons TCG (Ser), CGC (Arg), CGA (Arg), GCG (Ala), GTA(Val) and CCG(Pro) stand out as the most informative codons. As the classification algorithm treats all features as equals, such increased importance of certain codons might hint at underlying biological causes.

\section{Neural networks}

To confirm and potentially improve the results from the RF classifier, we also trained a neural network (NN) classifier on the same RSCU data. We applied the same leaveone-experiment-out cross-validation technique, meaning 19 models were trained, each time leaving out data from one experiment. Table 2 summarizes the results for the best-performing (according to ROC area) neural network hyperparameters at classification threshold 0.5 using the same measures as for random forests. The results at threshold 0.5 for $\mathrm{NN}$ approach look superior to the results of random forests when comparing F1 scores for the viral class. However, area under the ROC curve reveals that the two methods are of equivalent power. The micro-averaged area under the ROC curve for this best model is 0.790, whereas the best random forest configuration reaches 0.789 . Indeed compared to the random forests in Table 1 the neural networks in Table 2 seemed to trade off precision at the expense or recall, with the actual underlying power to discriminate (as measured by ROC area) staying the same.

Thus both random forest and neural network models are able to detect patterns in the RCSU values that are predictive of the virus or non-virus nature of the samples.

\section{Discussion}

We found that machine learning using RSCU can predict presence of viral contigs in metagenomic sequencing data. 


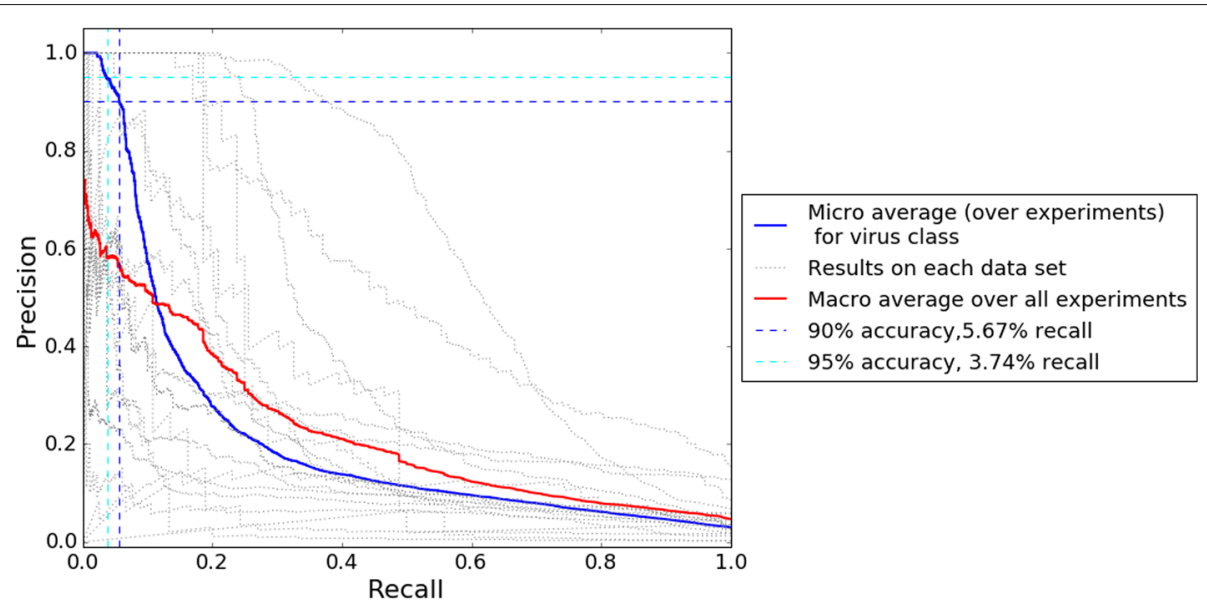

Fig. 5 The trade-off between precision and recall for virus vs. non-virus classification when changing the classification threshold. A sample is classified as virus only if $\mathrm{p}(\mathrm{virus})>$ threshold. Dotted lines depict the precision and recall for virus class at different thresholds for individual folds (i.e. metagenomics experiments) in LOEO cross validation. Blue line depicts the micro average across the experiments, red line illustrates macro average. The vertical and horizontal dashed lines exemplify what performance can be obtained when boosting precision at the expense of recall. Blue dashed line shows that at $90 \%$ accuracy we can obtain 5.67\% recall. Cyan dashed line shows that a threshold giving us $95 \%$ precision would yield $3.74 \%$ recall. The models yielding highest area under ROC curve are used for this graph

Firstly, we investigated the possibility to use RSCU values to predict viral origin of sequences originating from complete protein coding genes at NCBI Genbank (the data was obtained from http://www.kazusa.or.jp/codon/). Using 10-fold cross-validation on this dataset, random forest models achieved 0.996 area under ROC curve on validation data. However, the models trained on this dataset failed to generalize when tested on metagenomics data - yielding only 0.510 area under ROC. Additionally, we tested the metagenomics model on the Genbank

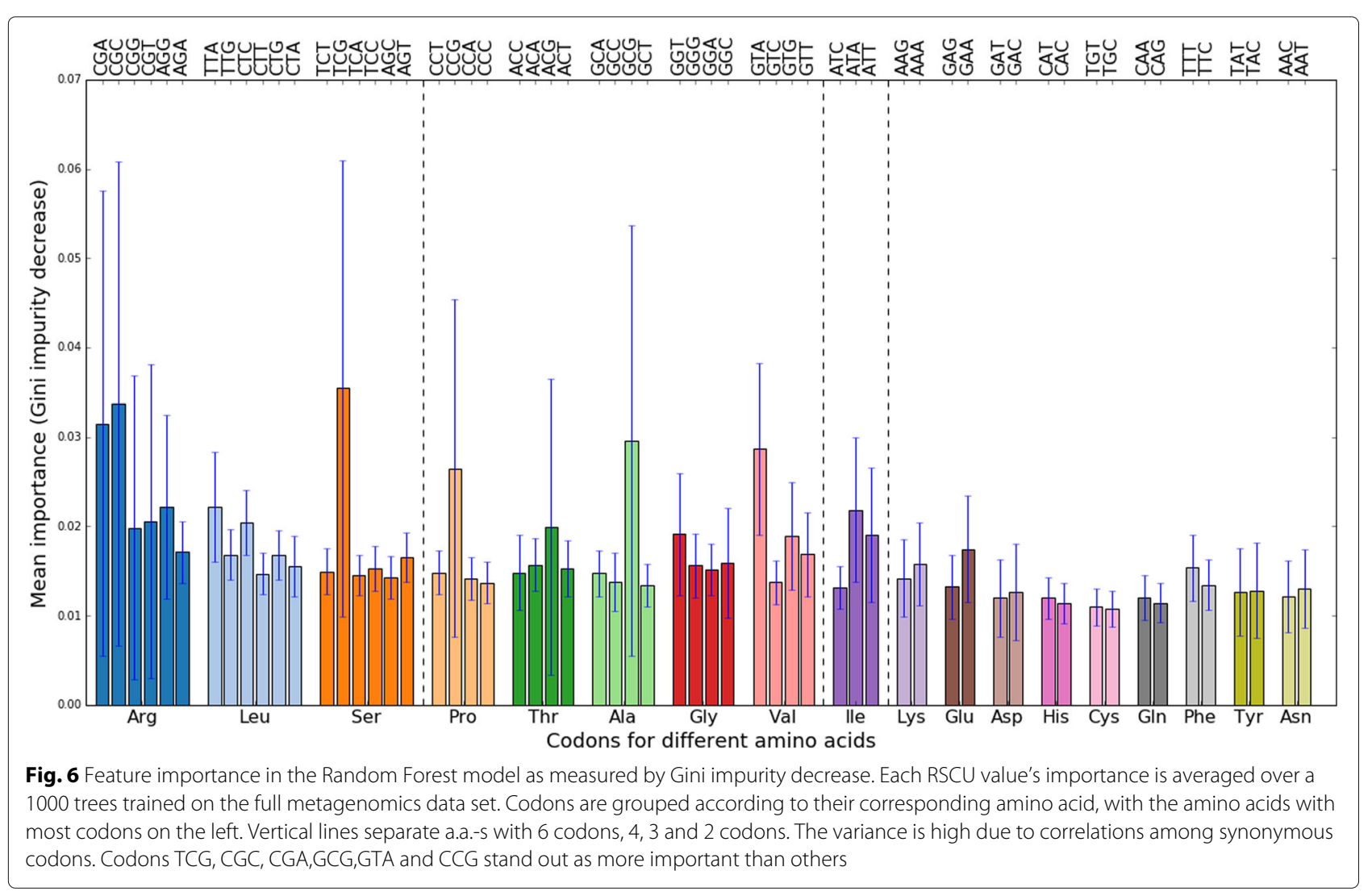


Table 2 Micro- and macro-average performance measures across 19 experiments using feed-forward neural networks

\begin{tabular}{lllll}
\hline Method & Class & Precision & Recall & F1-score \\
\hline Micro-average & Non virus & 0.97 & 1.00 & 0.99 \\
Micro-average & Virus & 0.69 & 0.13 & 0.21 \\
Macro-average & Non virus & 0.96 & 1.00 & 0.98 \\
Macro-average & Virus & 0.43 & 0.12 & 0.19 \\
\hline
\end{tabular}

The results are from the best model according to area under the ROC curve $\left(R O C_{\text {micro }}=0.790\right)$. This model used two 1024 units FC layers with Relu nonlinearity, 0.25 dropout rate and class_weight_power 0.25 (see Additional file 3). All networks were trained for 10 epochs, using Adam optimizer with $10 e-4$ initial learning rate that was multiplied with 0.95 after each epoch

dataset, but results were also close to random guess. This failure probably occurs because metagenomic dataset is very noisy compared to the clean data obtained from Genbank. Instead of complete genes, it contains shorter fragments, it includes non-coding ORFs and has many sources of possible errors in the pipeline. Considering the differences between the two datasets, it is a logical outcome that a model built on one dataset does not perform on the other. As our goal is to detect viral sequences specifically in metagenomics data (and not just generally demonstrate predictability using RSCU values), we concluded that we should also train the model on metagenomics data.

Using both Feed Forward Neural Network and Random Forest classification methods and metagenomics as training dataset, we show that RSCU can predict the viral nature of a sequence in metagenomic dataset. While the method rediscovers only a small proportion of the viral contigs we nevertheless consider this a significant result because this rediscovery was achieved based solely on RSCU values extracted from ORFs (most of ORFs are probably not actually genes) without any additional external knowledge - such as a sequence database. Having used such very high-level features this method has a chance of generalizing outside the space of "known sequences" that it was trained on. This means that the presented solution can be applied to sequences that other, more informed methods leave unclassified because they are not similar enough to the "known sequences" in the database. This information is beyond the information offered by other methods and it significantly narrows down the search space for the discovery of unknown viruses in metagenomic samples.

We also investigated which codons played a decisive role, employing the Random Forest feature importance analysis. RSCU values for six codons (TCG (Ser), CGC (Arg), CGA (Arg), GCG (Ala), GTA(Val) and CCG(Pro)) were the most influential in the classification model. In the human genome, none of these 6 codons are frequently used [39]. In our metagenomics datasets, the average RSCU values for the top two influential codons, TCG and CGC, in non-viral contigs were also quite low (0.39 and 0.53$)$, while in viral contigs they were more abundant $(0.60$ and 0.80$)$. A similar pattern is also followed by the other four most influential codons (see Additional file 6 for a figure depicting mean RSCU values in the two classes). This indicates that the most decisive codons for the algorithm were the ones, which were least commonly found in non-viral sequences. It also suggests that the frequency of usage of these particular codons is different in viral and non-viral genome, which in turn hints at different biological characteristics of viral sequences. Further research will be necessary to analyze this difference.

Metagenomics datasets generated by NGS technologies from human biospecimens are noisy and contain many potential errors. After human samples are sequenced, the Illumina machine provides a vast amount of fragmented 'reads' of DNA [40]. In order to reconstruct full genomes, de novo assembly algorithms are used, which introduces several types of errors, such as substitutions, insertions or deletions. These errors cause frame shifts in the potential coding regions that may greatly affect accuracy of RSCU values. Despite this highly noisy data (that comprised only $3 \%$ of viral sequences) our approach achieved 0.79 area under the ROC curve. In our bioinformatics pipeline, for the sequences that are classified as unknown by NCBI BLAST, the HMMER3 algorithm is applied. However, a large amount of sequences is still labeled as unknown where potential viral sequences might be hidden. Therefore, we propose that the machine learning models proposed in this work could be used as the third stage after BLAST and HMMER3.

De novo assembly for viral metagenomics is in its infancy and further improvements will most probably further enhance the predictive value of machine learning analysis of RCSU values for taxonomic classification.

\section{Conclusions}

The results of the present investigation indicate that simple counting statistics at the codon level and applying machine learning to RCSU values can provide important information in addition to conventional methods for taxonomic classification of sequences in metagenomic datasets. Future investigations should focus on a more flexible approach without pre-defined features (like RSCU values), such as training $1 \mathrm{D}$ convolutional neural networks on raw DNA sequence strings. This approach may have the potential to discover novel predictive features beyond codon usage and thus further improve the classification accuracy. 


\section{Additional files}

Additional file 1: Metadata of the samples used for this study. (XLSX $40 \mathrm{~kb}$ ) Additional file 2: Number of the assembled contigs classified into different taxonomy groups by BLAST. (XLSX $32 \mathrm{~kb}$ )

Additional file 3: Methods description in detail. (DOCX $19 \mathrm{~kb}$ ) Additional file 4: Results with different hyperparameters for random forest. (XLSX $13 \mathrm{~kb}$ )

Additional file 5: Results per experiment (random forest). (XLSX 32 kb)

Additional file 6: Figure summarizing mean RSCU values in the two classes. (PDF $64 \mathrm{~kb}$ )

Additional file 7: Counted RSCU values from metagenomic dataset. (TXT $59,594 \mathrm{~kb})$

\section{Abbreviations}

FFN: Feed-forward neural network; LOEO: Leave-one-experiment-out cross validation; NGS: Next generation sequencing; NN: Neural networks; ORFs: Open reading frames; RF: Random forest; ROC: Receiver operating characteristic curve; RSCU: Relative synonymous codon usage

\section{Acknowledgements}

ZB and JD acknowledge support from the Swedish Research Council and from the Nordic Academy for Advanced Studies (NordForsk) to the Nordic Information for Action eScience Center of Excellence.

A.T. and R.V. thank the financial support from the Estonian Research Council through the personal research grant PUT1476. This work was also supported by the Estonian Centre of Excellence in IT (EXCITE), funded by the European Regional Development Fund.

\section{Funding}

The study is supported by Swedish Research Council, the Nordic Academy for Advanced Studies (NordForsk) to the Nordic Information for Action eScience Center of Excellence and Estonian Research Council.

\section{Availability of data and materials}

Counted RSCU values on metagenomic contigs (training dataset) is provided as Additional file 7. The code to compute RSCU values from Fasta files on Apache Spark is available here (https://github.com/NGSeq/ViraPipe).

\section{Authors' contributions}

ZB and AT implemented and performed all computational work and wrote the main manuscript text. PB analysed the dataset and wrote the main manuscript text. RV and JD directed the project and wrote the main manuscript text. All authors reviewed the manuscript. All authors read and approved the final manuscript.

\section{Ethics approval and consent to participate}

Our study is based on re-analysis of a series of previous studies on metagenomics sequencing, analysed with the bioinformatics pipeline that was most up-to-date at that time. The studies had the following Ethical Review Board (ERB) permissions: 2011/1026-31/4; 2012/1028/32; 53/2005; 612/2008; LU574-03; 104/2006; R13149, 2/2014; 2011-198-31M and 12/780-32. In the Swedish system, the Ethical Review Board (ERB) is appointed by government and chaired by a senior judge. The ERB has the authority to specify the demands on information and consent and the ERB decisions were carefully followed and our study is thus in accordance with the Declaration of Helsinki.

\section{Consent for publication}

Not applicable.

\section{Competing interests}

The authors declare that they have no competing interests.

\section{Publisher's Note}

Springer Nature remains neutral with regard to jurisdictional claims in published maps and institutional affiliations.

\section{Author details}

${ }^{1}$ Dept. of Laboratory Medicine, Karolinska Institutet, F46, Karolinska University Hospital Huddinge, Stockholm, Sweden. ${ }^{2}$ Institute of Computer Science, University of Tartu, Tartu, Estonia. ${ }^{3}$ Interdisciplinary Centre for Mathematical and Computational Modelling, University of Warsaw, Warsaw, Poland.

Received: 26 September 2017 Accepted: 28 August 2018

Published online: 24 September 2018

\section{References}

1. Wylie KM, Weinstock GM, Storch GA. Emerging view of the human virome. Transl Res. 2012;160(4):283-90

2. Lecuit M, Eloit M. The human virome: new tools and concepts. Trends Microbiol. 2013;21(10):510-5.

3. Wylie KM, Mihindukulasuriya KA, Sodergren E, Weinstock GM, Storch GA. Sequence analysis of the human virome in febrile and afebrile children. PloS ONE. 2012;7(6):27735.

4. Willner D, Furlan M, Haynes M, Schmieder R, Angly FE, Silva J, Tammadoni S, Nosrat B, Conrad D, Rohwer F. Metagenomic analysis of respiratory tract dna viral communities in cystic fibrosis and non-cystic fibrosis individuals. PloS ONE. 2009;4(10):7370.

5. Bzhalava D, Ekström J, Lysholm F, Hultin E, Faust H, Persson B, Lehtinen M, de Villiers E-M, Dillner J. Phylogenetically diverse tt virus viremia among pregnant women. Virology. 2012;432(2):427-34

6. Bzhalava D, Hultin E, Arroyo Mühr LS, Ekström J, Lehtinen M, de Villiers E-M, Dillner J. Viremia during pregnancy and risk of childhood leukemia and lymphomas in the offspring: Nested case-control study. Int J Cancer. 2016;138(9):2212-20.

7. Bzhalava D, Johansson H, Ekström J, Faust H, Möller B, Eklund C, Nordin P, Stenquist B, Paoli J, Persson B, et al. Unbiased approach for virus detection in skin lesions. PLoS ONE. 2013;8(6):65953.

8. Bzhalava D, Mühr LS, Lagheden C, Ekström J, Forslund O, Dillner J, et al. Deep sequencing extends the diversity of human papillomaviruses in human skin. Sci Rep. 2014;4:5807.

9. Ekström J, Bzhalava D, Svenback D, Forslund O, Dillner J. High throughput sequencing reveals diversity of human papillomaviruses in cutaneous lesions. Int J Cancer. 2011:129(11):2643-50.

10. Feng $H$, Shuda M, Chang $Y$, Moore PS. Clonal integration of a polyomavirus in human merkel cell carcinoma. Science. 2008;319(5866): 1096-100.

11. Meiring TL, Salimo AT, Coetzee B, Maree HJ, Moodley J, Hitzeroth II, Freeborough M-J, Rybicki EP, Williamson A-L. Next-generation sequencing of cervical dna detects human papillomavirus types not detected by commercial kits. Virol J. 2012:9(1):164.

12. Foulongne V, Sauvage V, Hebert C, Dereure O, Cheval J, Gouilh MA Pariente K, Segondy M, Burguière A, Manuguerra J-C, et al. Human skin microbiota: high diversity of dna viruses identified on the human skin by high throughput sequencing. PloS ONE. 2012;7(6):38499.

13. Towner JS, Sealy TK, Khristova ML, Albariño CG, Conlan S, Reeder SA, Quan P-L, Lipkin WI, Downing R, Tappero JW, et al. Newly discovered ebola virus associated with hemorrhagic fever outbreak in uganda. PLoS Pathog. 2008:4(11):1000212.

14. Willner D, Haynes MR, Furlan M, Hanson N, Kirby B, Lim YW, Rainey PB, Schmieder R, Youle M, Conrad D, et al. Case studies of the spatial heterogeneity of dna viruses in the cystic fibrosis lung. Am J Respir Cell Mol Biol. 2012;46(2):127-31.

15. Johansson H, Bzhalava D, Ekström J, Hultin E, Dillner J, Forslund O. Metagenomic sequencing of "hpv-negative" condylomas detects novel putative hpv types. Virology. 2013;440(1):1-7.

16. Labonté JM, Suttle CA. Previously unknown and highly divergent ssdna viruses populate the oceans. ISME J. 2013:7(11):2169.

17. Thomas T, Gilbert J, Meyer F. Metagenomics-a guide from sampling to data analysis. Microb Inform Experimentation. 2012;2(1):3.

18. Skewes-Cox P, Sharpton TJ, Pollard KS, DeRisi JL. Profile hidden markov models for the detection of viruses within metagenomic sequence data. PLOS ONE. 2014;9(8):105067.

19. Bzhalava Z, Hultin E, Dillner J. Extension of the viral ecology in humans using viral profile hidden markov models. Plos ONE. 2018;13(1):1-12.

20. Shin YC, Bischof GF, Lauer WA, Desrosiers RC. Importance of codon usage for the temporal regulation of viral gene expression. Proc Natl Acad Sci. 2015;112(45):14030-5 
21. Athey J, Alexaki A, Osipova E, Rostovtsev A, Santana-Quintero LV, Katneni U, Simonyan V, Kimchi-Sarfaty C. A new and updated resource for codon usage tables. BMC Bioinformatics. 2017;18(1):391.

22. Smelov V, Bzhalava D, Mühr LSA, Eklund C, Komyakov B, Gorelov A, Dillner J, Hultin E. Detection of dna viruses in prostate cancer. Sci Rep. 2016;6:25235

23. Mühr LSA, Bzhalava D, Lagheden C, Eklund C, Johansson H, Forslund O, Dillner J, Hultin E. Does human papillomavirus-negative condylomata exist? Virology. 2015;485:283-8.

24. Arroyo Mühr LS, Hultin E, Bzhalava D, Eklund C, Lagheden C, Ekström J, Johansson H, Forslund O, Dillner J. Human papillomavirus type 197 is commonly present in skin tumors. Int J Cancer. 2015;136(11):2546-55.

25. Bzhalava D, Dillner J. Bioinformatics for viral metagenomics. J Data Min Genom Proteomics. 2013;4:134.

26. Li H, Durbin R. Fast and accurate long-read alignment with burrows-wheeler transform. Bioinformatics. 2010;26(5):589-95.

27. Grabherr MG, Haas BJ, Yassour M, Levin JZ, Thompson DA, Amit I, Adiconis X, Fan L, Raychowdhury R, Zeng Q, Chen Z, et al. Trinity: reconstructing a full-length transcriptome without a genome from rna-seq data. Nat Biotechnol. 2011;29:644-52.

28. Luo R, Liu B, Xie Y, Li Z, Huang W, Yuan J, He G, Chen Y, Pan Q, Liu Y, Tang J, Wu G, Zhang H, et al. Soapdenovo2: an empirically improved memory-efficient short-read de novo assembler. GigaScience. 2012;1 (1):1-6

29. Peng Y, Leung HCM, Yiu SM, Chin FYL. Idba-ud: a de novo assembler for single-cell and metagenomic sequencing data with highly uneven depth. Bioinformatics. 2012;28(11):1420-8.

30. Nowicki M, Bzhalava D, Bała P. Massively parallel implementation of sequence alignment with basic local alignment search tool using parallel computing in java library. J Comput Biol. 2018;25(8):871-81.

31. Sharp PM, Tuohy TM, Mosurski KR. Codon usage in yeast: cluster analysis clearly differentiates highly and lowly expressed genes. Nucleic Acids Res. 1986;14(13):5125-43.

32. Fu L, Niu B, Zhu Z, Wu S, LiW. Cd-hit: accelerated for clustering the next-generation sequencing data. Bioinformatics. 2012;28(23):3150-2.

33. Van Asch V. Macro-and micro-averaged evaluation measures. Tech Rep. 2013.

34. Breiman L. Random forests. Mach Learn. 2001;45(1):5-32.

35. Pedregosa F, Varoquaux G, Gramfort A, Michel V, Thirion B, Grisel O, Blondel M, Prettenhofer P, Weiss R, Dubourg V, et al. Scikit-learn: Machine learning in python. J Mach Learn Res. 2011;12(Oct):2825-30

36. Strobl C, Boulesteix A-L, Kneib T, Augustin T, Zeileis A. Conditional variable importance for random forests. BMC Bioinformatics. 2008;9(1):307

37. Archer KJ, Kimes RV. Empirical characterization of random forest variable importance measures. Comput Stat Data Anal. 2008;52(4):2249-60.

38. Bishop CM. Neural networks for pattern recognition. Oxford: Oxford University Press; 1995

39. Castro-Chavez F. Most used codons per amino acid and per genome in the code of man compared to other organisms according to the rotating circular genetic code. NeuroQuantology Interdiscip J Neurosci Quantum Phys. 2011:9(4):500.

40. Henson J, Tischler G, Ning Z. Next-generation sequencing and large genome assemblies. Pharmacogenomics. 2012;13(8):901-15.

Ready to submit your research? Choose BMC and benefit from:

- fast, convenient online submission

- thorough peer review by experienced researchers in your field

- rapid publication on acceptance

- support for research data, including large and complex data types

- gold Open Access which fosters wider collaboration and increased citations

- maximum visibility for your research: over $100 \mathrm{M}$ website views per year

At BMC, research is always in progress.

Learn more biomedcentral.com/submissions 